(C) 2015 IEEE. Personal use of this material is permitted. Permission from IEEE must be obtained for all other uses, in any current or future media, including reprinting/republishing this material for advertising or promotional purposes, creating new collective works, for resale or redistribution to servers or lists, or reuse of any copyrighted component of this work in other works.

This is the Author's Accepted Manuscript version of a paper published in: Communications, Signal Processing, and their Applications (ICCSPA), 2015 International Conference on, by IEEE on 17 February 2015.

The full citation of the original IEEE publication is:

Arshad, Kamran, "LTE system level performance in the presence of CQI feedback uplink delay and mobility," Communications, Signal Processing, and their Applications (ICCSPA), 2015 International Conference on, pp.1,5, 17-19 Feb. 2015 doi: 10.1109/ICCSPA.2015.7081294

The final publication is available at IEEE via: http://dx.doi.org/10.1109/ICCSPA.2015.7081294 


\title{
LTE System Level Performance in the Presence of CQI Feedback Uplink Delay and Mobility
}

\author{
Kamran Arshad \\ Mobile and Wireless Communications Research Labortory \\ Electrical, Electronic and Computer Engineering \\ University of Greenwich, United Kingdom \\ Email: k.arshad@greenwich.ac.uk
}

\section{ABSTRACT}

Long Term Evolution (LTE) is a promising technology for the wireless and mobile communication systems and is being commercially launched in various countries of the world. The Third Generation of Partnership Project (3GPP), through design and optimisation of new radio access techniques, is further developing the future LTE-Advanced technology to remain on the forefront of wireless technologies. However, it is still challenging in LTE to provide the desired quality of service to the users particularly in the presence of high mobility and uplink feedback delay. In this paper, the impact of Channel Quality Indicator (CQI) uplink feedback delay on the overall network performance under different scheduling algorithms and mobility patterns is discussed. We study average user equipment (UE) throughput, average cell-edge UE throughput and average cell throughput under mobility and conclude that for an efficient LTE-Advanced scheduling algorithm, UE speed and CQI feedback delay must be taken into account [1].

\section{INTRODUCTION}

LTE was standardised by 3GPP, initially in release 8 (December 2008), now commercially available in various countries all around the world. LTE specifications are now stable and several enhancements were added in subsequent 3GPP releases (i.e. R9 - R13). LTE and LTE-Advanced specifications are targeting to achieve higher peak data rates, higher spectral efficiency, lower latency and all IP based optimised network [2]. Higher data rate, and spectral efficiency is achieved by using flexible, scalable carrier bandwidths ranging from $1.4 \mathrm{MHz}$ up to $100 \mathrm{MHz}$, Orthogonal Frequency Division Multiple Access (OFDMA) in downlink, Adaptive Modulation and Coding Scheme (A-MCS) and Multiple Input Multiple Output (MIMO).

In order to achieve target data rate or throughput, eNodeB selects the best MCS for each sub-carrier depending on the channel state information (CSI). At the UE, a module is responsible for processing the CSI information, for instance, estimating sub-carriers received Signal to Interference Noise Ratio (SINR) to obtain a Block Error Rate (BLER) estimate under the assumption of a particular channel model. An offline lookup table can be used to map this information into a higher rate CQI value that can specify a $10 \%$ BLER target [3]. Such static BLER curves under a specific channel model can be generated either by link level simulations or mathematical models [4]. Finally, the selected values are sent to the eNodeB as a CQI feedback vector. Based on the received CQI, eNodeB perform resource scheduling and MCS selection.

Packet level scheduling is one of the core functionality of LTE and plays an important role in the optimisation of the network. A scheduling scheme can be designed to allocate each UE a portion of the available resources i.e. resource blocks (RBs). For an efficient scheduling, ideally each UE needs to send periodically a set (one for each sub-band) of CQI values to the eNodeB. However, this would result a tremendous uplink control signalling overhead. Further, in practice, delays of several transmission time intervals (TTIs) in the reception of CQI feedback at eNodeB may exist that causes severe performance degradation. In fast fading channels, the CQI at the scheduling instant greatly deviates the instant when CQI was generated and hence negatively affect the accuracy of MCS selection [5].

Several CQI feedback reporting and compression schemes have been proposed in the literature [6]. The degradation of system performance due to the delayed CQI feedback was analysed in [7], [8]. Depending on the scheduling scheme, an eNodeB may select the UE with highest sub-channel CQI values to maximise the system throughput. As 3GPP did not specify scheduling mechanisms for both uplink and downlink, a large number of scheduling algorithms have been proposed in the literature, for more details reader may refer to [9].

There are several technical challenges that will need to be addressed in the future releases of 3GPP LTE (i.e. LTEAdvanced). In this paper, we show that the impact of CQI feedback delay becomes worst as UE speed increases and hence design of a scheduling algorithm incorporating UE speed for future LTE releases is an important task. The aim of this paper is to investigate the impact of UE speed on CQI feedback delay under different scheduling algorithms proposed in the literature, more specifically, we consider best cqi (Bcqi), round robin $(R R)$ and proportional fair $(P F)$ algorithms [9]. The simulations were performed using the LTE system level simulator discussed in [3]. The outcome of this study will provide a guideline for the design of scheduling algorithms for the future LTE-Advanced systems.

The remainder of this paper is organised as follows: section II describes the preliminaries: overview of LTE downlink, 
CQI measurements and feedback. This is followed by a brief overview of LTE schedulers in section III. Simulation details, results and discussion are provided in section IV. Finally, section $\mathrm{V}$ concludes this paper.

\section{SYSTEM MOdEL}

\section{A. LTE Downlink Overview}

The 3GPP LTE standard supports both frequency division duplex (FDD) and time division duplex (TDD) and can be deployed over a wider range of bandwidth $(1.4 \mathrm{MHz}, 3 \mathrm{MHz}$, $5 \mathrm{MHz}, 10 \mathrm{MHz}$ and $20 \mathrm{MHz}$ ). In this paper, we consider only FDD with $20 \mathrm{MHz}$ bandwidth for the sake of simplicity and similar conclusions can be drawn for other bandwidth as well. In OFDMA based LTE downlink, the minimum resource that can be assigned to a user is referred as a RB. A RB is defined as a group of 12 sub-carriers (or $180 \mathrm{KHz}$ ) in the frequency domain and a half TTI i.e. $0.5 \mathrm{~ms}$ slot in the time domain (1 TTI $=2 \times 0.5 \mathrm{~ms}$ slot). In each TTI, a radio frequency carrier consists of any number of RBs ranging from a minimum of 6 to a maximum of $110 \mathrm{RB}$ that corresponds transmission bandwidth from $1.4 \mathrm{MHz}$ to $20 \mathrm{MHz}$ with a granularity of $15 \mathrm{kHz}$ [4]. In LTE, scheduling is done on a TTI basis, hence a RB is assigned to a user for two consecutive time slots. In a time slot, 7 OFDM symbols are transmitted hence if $1 \mathrm{RB}$ is assigned to a user for one TTI, a total of 168 OFDM subcarriers transmits. The physical layer (PHY) receives user data from the MAC layer in the form of an entity called transport block (TB).

\section{B. CQI Measurements}

UE makes measurement of SINR at each RB and then convert the estimated value of SINR into a corresponding CQI for which the received TB BLER shall not exceed 10\% [4]. In this paper, we assume a multi-cell multi-user (MC-MU) scenario where a cell is surrounded by a number of interfering cells, say $\varphi_{\text {int }}$. The received SINR at $k^{\text {th }}$ sub-carrier can be written as

$$
\gamma_{k}=\frac{\frac{\left|h_{0, k}\right|^{2}}{L_{0, k}} P_{0, k}}{\sum_{i=1}^{\varphi_{\text {int }}} \frac{\left|h_{i, k}\right|^{2}}{L_{i, k}} P_{i, k}+\zeta_{w}^{2}}
$$

where $P_{0}$ is the received power from UE own eNodeB, $P_{i}$ is the received power from $i^{\text {th }}$ interfering eNodeB, $L$ is the path loss, $h$ is the complex channel gain and $\zeta_{w}^{2}$ is the noise power. We assume an urban environment based on 3GPP TS 36.942 path loss model [10] and ITU Vehicular-B channel model is used for the generation of channel coefficients.

Due to the channel frequency selectivity, received SINR on RBs differs from each other. In order to estimate CQI, SINRs on different RBs must be mapped into an effective SINR, obtained from mapping the sub-carrier SINR to an AWNG equivalent SINR. The BLER of the effective SINR, $\gamma_{\text {eff }}$ in AWGN channel should match the original TB BLER as

$$
p_{e}\left(\gamma_{\text {eff }}\right)=p_{e}\left(\gamma_{k}, k \in \mathcal{C}\{b\}\right)
$$

where $\mathcal{C}\{b\}$ is the set of RBs in sub-band $b$.
The exponential effective signal to interference and noise ratio mapping (EESM) is used in the simulations to obtain the $\gamma_{\text {eff }}$, which can be used to map the TB BLER from either mathematical models or link level simulations.

$$
\gamma_{\text {eff }}=-\beta \cdot \log \left(\frac{1}{N} \sum_{k \in N} \exp \left(-\frac{\gamma_{k}}{\beta}\right)\right)
$$

where $N$ is the number of sub-carriers and $\beta$ is a calibration parameter to fit the compression function in (3) to AWGN BLER curves. The $\gamma_{\text {eff }}$ is finally mapped by means of an AWGN BLER curve of the corresponding MCS to a BLER value. Fig. 1 shows the BLER curve for each CQI value in AWGN channel and each CQI value represents a unique combination of modulation scheme and coding rate. From Fig. 1, the CQI feedback value can be obtained using a simple step function, $\mathcal{Q}=f\left(\gamma_{\text {eff, dB }}\right)$ as shown in Fig. 2.

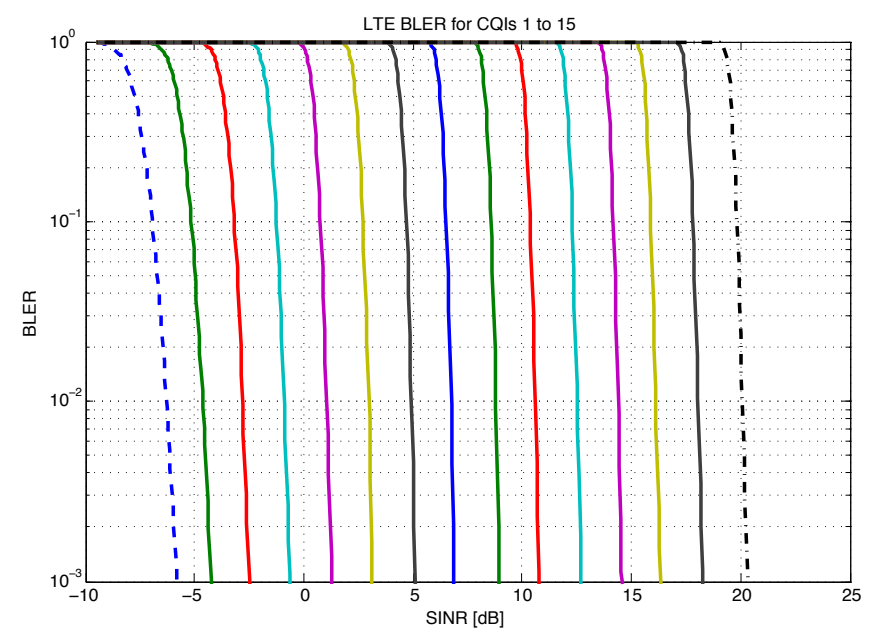

Fig. 1. BLER curves for CQI values from CQI 1 (leftmost, dash) to CQI 15 (rightmost, dash-dot) for $1.8 \mathrm{GHz}$ carrier frequency, $20 \mathrm{MHz}$ channel bandwidth and 1000 TTI simulation time

\section{CQI Feedback}

According to LTE standard, each UE measures downlink channel quality in terms of estimated SINR. The SINR-to-CQI mapping is realised and an appropriate CQI value is selected as explained in section II-B. The obtained CQI values, then, reported back to eNodeB using a CQI reporting scheme on a particular control channel (HS-DPCCH) which may arrive at eNode with a certain delay. The feedback delay time comprises of processing time, transmission time, and time taken when waiting for a scheduling slot [6]. The delay in the reception of CQI feedback at eNodeB may cause significant performance degradation. Depending on how quickly scheduler assigns radio resources to the users, the delay may be about 1 to 4 TTI (each TTI is $1 \mathrm{~ms}$ ).

\section{OVERVIEW OF LTE SCHEDULERS}

Scheduling is a process of dynamically allocating RBs among UE based on scheduling algorithm and the received CQI values. The scheduler is located at an eNodeB and decides 


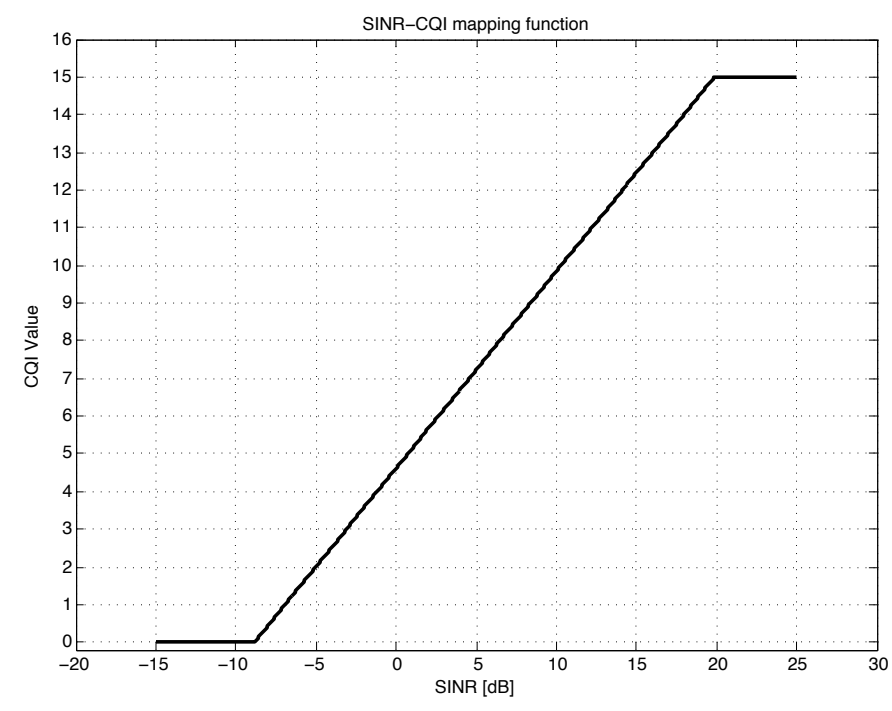

Fig. 2. SINR to CQI mapping function consisting of $10 \%$ BLER points from Fig. 1

which users to be scheduled and which RBs need to be allocated in a given TTI by taking into account various factors such as throughput, spectral efficiency, fairness etc. [9].

\section{A. best cqi (Bcqi) scheduler}

In this scheduling strategy, the radio resources are assigned to the users with best channel conditions (alternatively, users with higher values of CQI). In Bcqi the channel selected for transmission have high quality and with appropriate MCS, a higher throughput or higher system capacity can be achieved. The Bcqi increases cell capacity at the expense of fairness, as UEs with bad channel conditions (e.g. cell edge UE) are unlikely to be scheduled. Mathematically,

$$
k=\arg \max _{i} R_{i}
$$

where $R_{i}$ is the instantaneous data rate of $i^{\text {th }}$ user.

\section{B. Round Robin (RR) scheduler}

In this scheduling strategy, UE's are assigned RBs in turn one after another without taking into consideration the instantaneous channel conditions (or CQI values). Round robin scheduling is a fair scheduling scheme in a sense that same amount of radio resources (i.e. RB) are given to all users. Cell edge users with worst channel conditions get an equal share of radio resources, however, $R R$ is not fair in a sense of providing the same quality to all users. As $R R$ scheduling does not take into account the instantaneous channel conditions or CQI, the overall system throughput is lower.

\section{Proportional Fair $(P F)$ scheduler}

A $P F$ scheduler operates in between the $B c q i$ and $R R$ scheduler, i.e. taken into account the instantaneous channel conditions as much as possible while still satisfy some degree of fairness among users. In this strategy, the RBs are assigned
TABLE I

SIMULATION PARAMETERS

\begin{tabular}{l|c}
\hline Simulation Parameter & Value \\
\hline Carrier frequency & $1.8 \mathrm{GHz}$ \\
Bandwidth & $20 \mathrm{MHz}$ \\
Simulation scenario & SISO, Macro cell \\
Simulation time & $1000 \mathrm{TTI}$ \\
TTI length & $1 \mathrm{~ms}$ \\
Number of cells & 19 tri-sector eNodeBs \\
UE per eNodeB per sector & 100 \\
Path loss model & TS 36.942, urban $[10]$ \\
Std. deviation of shadow fading & $10 \mathrm{~dB}$ \\
eNodeB Tx Power & $40 \mathrm{~dB}$ \\
SINR averaging & EESM [3] \\
FFT size & 2048 \\
Data sub-carriers & 1200 \\
Cyclic Prefix & Normal [4] \\
Sampling frequency & $30.72 \mathrm{MHz}$ \\
Uplink feedback delay & $\{0,1,2,3,4\} \mathrm{TTI}$ \\
UE speed & $\{5,30,70,120\} \mathrm{Km} / \mathrm{Hr}$ \\
\hline
\end{tabular}

to the UE with relatively higher CQI i.e. at each time instant the $k^{\text {th }} \mathrm{UE}$ is selected as

$$
k=\arg \max _{i} \frac{R_{i}}{\overline{R_{i}}}
$$

where $\overline{R_{i}}$ is the average data rate of the $i^{\text {th }}$ UE.

\section{Simulation Results and Discussion}

We assume an OFDMA based downlink MC-MU scenario, consisting of 19 cells with identical 3 sector eNodeB's. The UE are uniformly distributed within each eNodeB sector. The wireless link attenuation consists of (1) a deterministic path loss urban model (2) a zero mean log-normally distributed shadow fading, and (3) a small scale Rayleigh fading. In all simulations, we assume ITU Vehicular-B power delay profile, as it is more realistic when users are moving at higher speeds. We consider a full buffer traffic model in our simulations that is there is always data available for every user. Other simulation parameters are summarised in Table I.

In this paper, system level performance is evaluated in terms of the average UE throughput, average cell-edge UE throughput and average cell throughput under the following scenarios: very low mobility $(5 \mathrm{Km} / \mathrm{Hr})$, average mobility $(30 \mathrm{Km} / \mathrm{Hr})$, high mobility $(70 \mathrm{Km} / \mathrm{Hr})$ and very high mobility $(120 \mathrm{Km} / \mathrm{Hr})$. For each scenario, we consider all three schedulers explained in section III. Average UE throughput for $B c q i, R R$ and $P F$ scheduler for different CQI feedback uplink delay is shown in Fig. 3, Fig. 4 and Fig. 5 respectively. The general trend is that the average user throughput decreases with CQI feedback delay. It is shown from Fig. 3 - Fig. 5 that at very low UE speed the average UE throughput is less affected by the CQI feedback delay. This is due to the fact that UE experience slow varying channel response at very low speed (i.e. $5 \mathrm{Km} / \mathrm{Hr}$ ) and hence delay has no significant impact (channel response is relatively same in subsequent TTI) 
TABLE II

Average Throughput of Cell Edge Users (MBPs) FOR $R R$ ALGORITHM

\begin{tabular}{c|c|c|c|c}
\hline Delay (in TTI) & $\mathbf{5} \mathbf{~ K m} / \mathbf{H r}$ & $\mathbf{3 0} \mathbf{~ K m} / \mathbf{H r}$ & $\mathbf{7 0} \mathbf{~ K m} / \mathbf{H r}$ & $\mathbf{1 2 0} \mathbf{~} \mathbf{m} / \mathbf{H r}$ \\
\hline 0 & 0.02 & 0.02 & 0.02 & 0.02 \\
1 & 0.01 & 0.01 & 0.01 & 0.00 \\
2 & 0.01 & 0.01 & 0.00 & 0.00 \\
3 & 0.01 & 0.01 & 0.00 & 0.00 \\
4 & 0.01 & 0.00 & 0.00 & 0.00 \\
\hline
\end{tabular}

TABLE III

Average Throughput of Cell Edge Users (MbPs) For $P F$ ALGORITHM

\begin{tabular}{c|c|c|c|c}
\hline Delay (in TTI) & $\mathbf{5} \mathbf{~} \mathbf{m} / \mathbf{H r}$ & $\mathbf{3 0} \mathbf{~} \mathbf{m} / \mathbf{H r}$ & $\mathbf{7 0} \mathbf{~ K m} / \mathbf{H r}$ & $\mathbf{1 2 0} \mathbf{~} \mathbf{m} / \mathbf{H r}$ \\
\hline 0 & 0.14 & 0.14 & 0.14 & 0.14 \\
1 & 0.11 & 0.09 & 0.03 & 0.01 \\
2 & 0.10 & 0.04 & 0.01 & 0.01 \\
3 & 0.09 & 0.02 & 0.01 & 0.00 \\
4 & 0.08 & 0.01 & 0.00 & 0.00 \\
\hline
\end{tabular}

on the users average throughput. Very high UE speed (i.e. $120 \mathrm{Km} / \mathrm{Hr}$ ) means faster temporal changes in the channel and hence even at very low delay average UE throughput is lowest and afterwards remain unaffected by the delay. For average and fast UE speed, it is clear how the average UE throughput decreases with the increase in delay. Similar trends are observed for average cell throughput as shown in Fig. 6, Fig. 7 and Fig. 8.

We consider three scheduling algorithms in our simulations: best cqi, round robin and proportional fair. It is shown in Fig. 3 and Fig. 6 that Bcqi gives superior performance in terms of average UE throughput and average cell throughput compare to other scheduling algorithms. This is an expected result as Bcqi schedules the users with the best channel conditions. Higher throughput of Bqi translates into the fact that the distribution of resources among users is not fair. While on the other hand, $R R$ and $P F$ average throughput is low (compared to Bcqi) but distribution of resources among users is fair, as in this scheduling algorithm cell-edge users are also served (see Fig. 4 and Fig. 5).

Table II and Table III show the average throughput of cell edge users in Mbps for $R R$ and $P F$ respectively. For $B c q i$ the average throughput of cell edge users is always zero irrespective of mobility. It is clear from Table II and Table III that both $R R$ and $P F$ algorithms serve all users irrespective of their channel conditions (i.e. CQI values). As a result, the average UE and cell throughout degrades but fairness among users increases. The $P F$ scheduling algorithm tries to balance throughput and fairness and in order to increase average throughput it assigns more resources to users with better channel conditions (i.e. higher CQI). The $P F$ algorithm is intelligently assign resources to users, providing a level of fairness among users while at the same time providing minimum quality of service to all users [9].

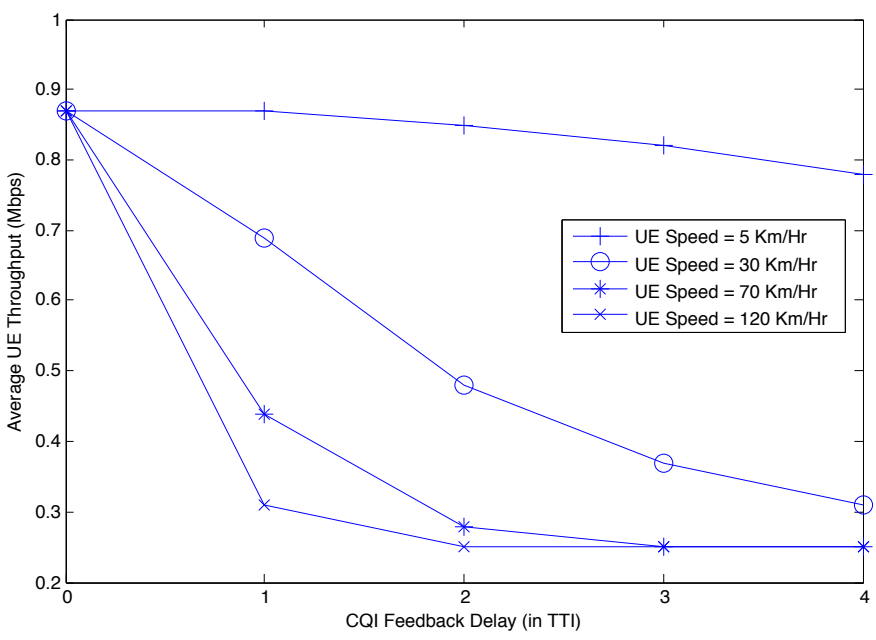

Fig. 3. Average UE Throughput versus Feedback delay for best cqi (Bcqi) scheduler

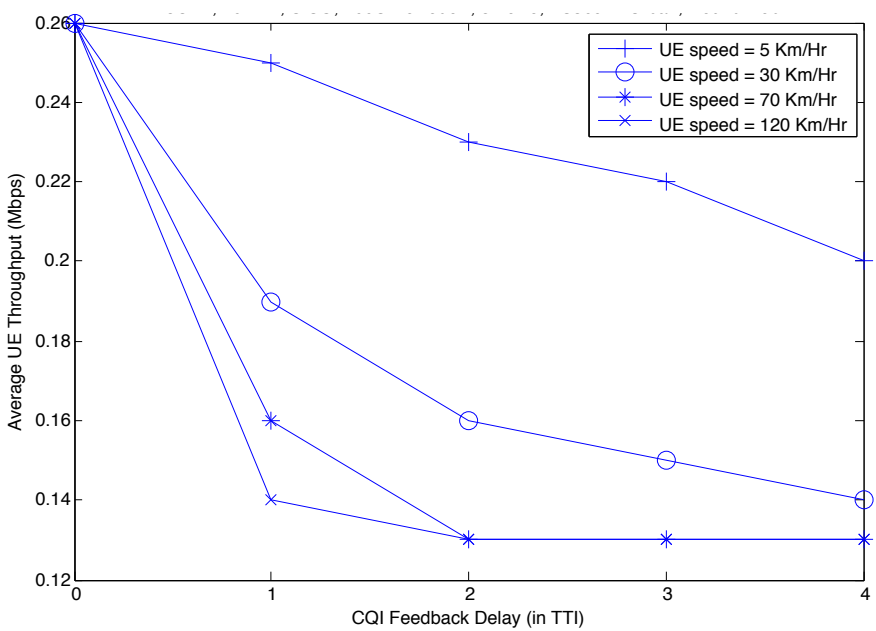

Fig. 4. Average UE Throughput versus Feedback delay for round robin $(R R)$ scheduler

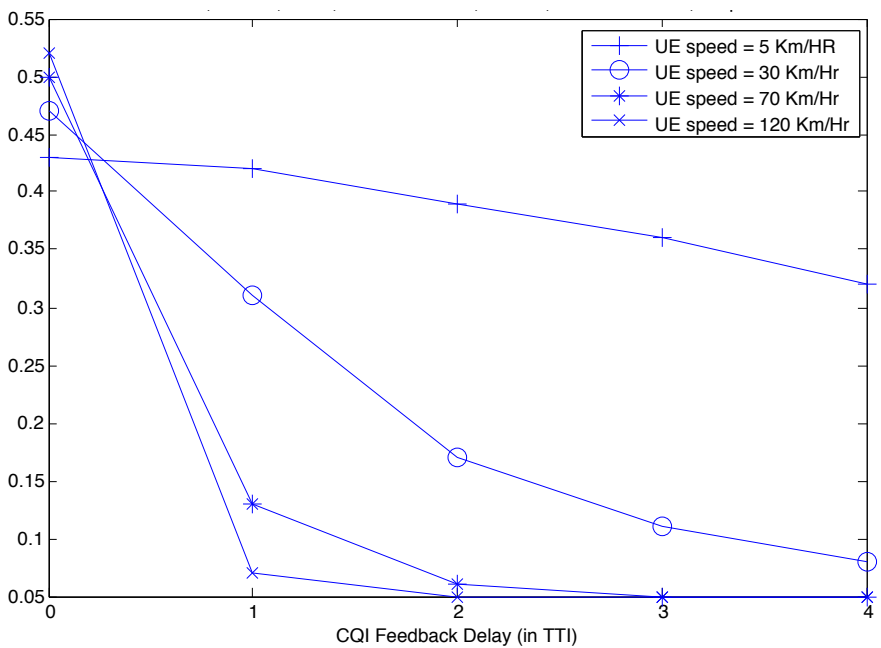

Fig. 5. Average UE Throughput versus Feedback delay for proportional fair $(P F)$ scheduler 


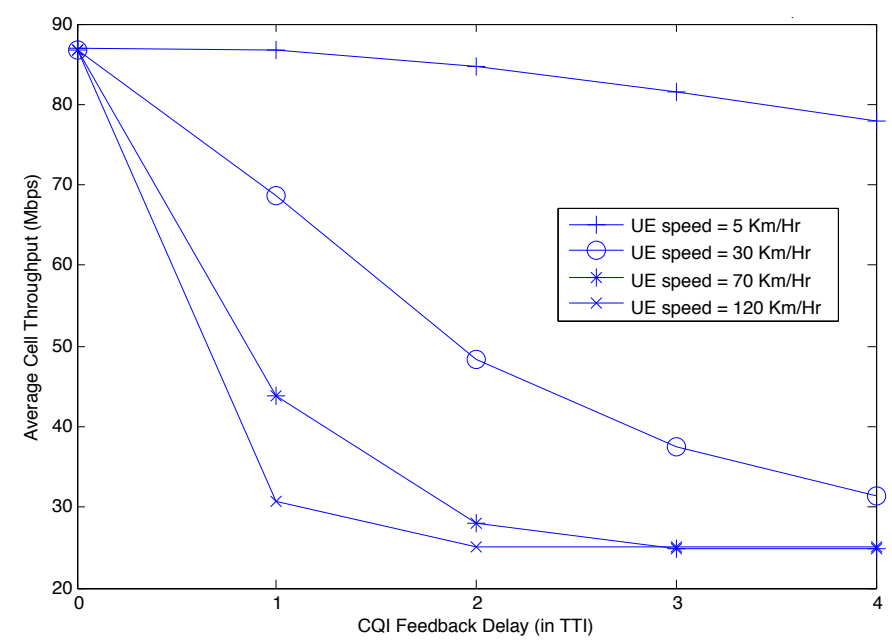

Fig. 6. Average Cell Throughput versus Feedback delay for best cqi (Bcqi) scheduler

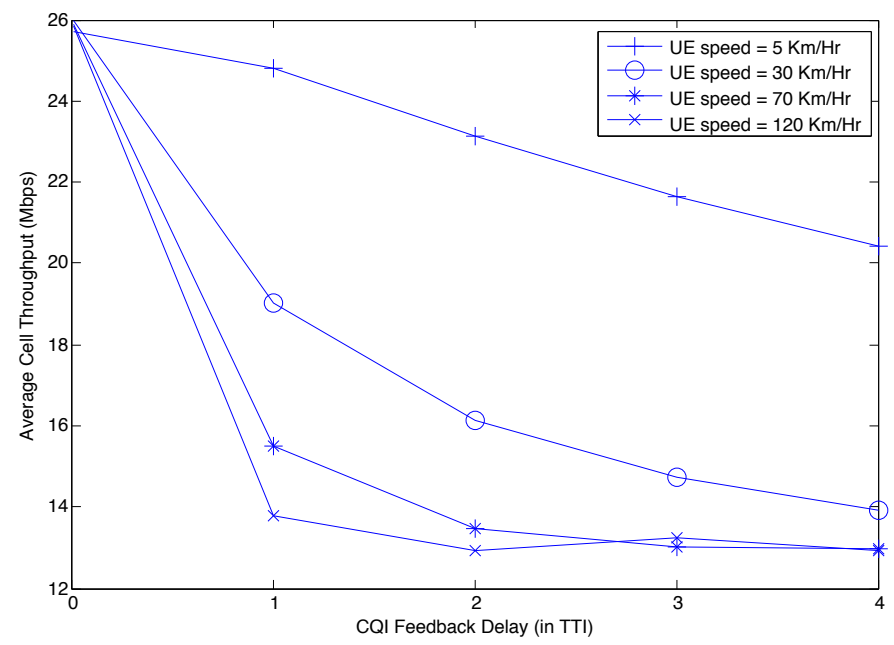

Fig. 7. Average Cell Throughput versus Feedback delay for round robin (RR) scheduler

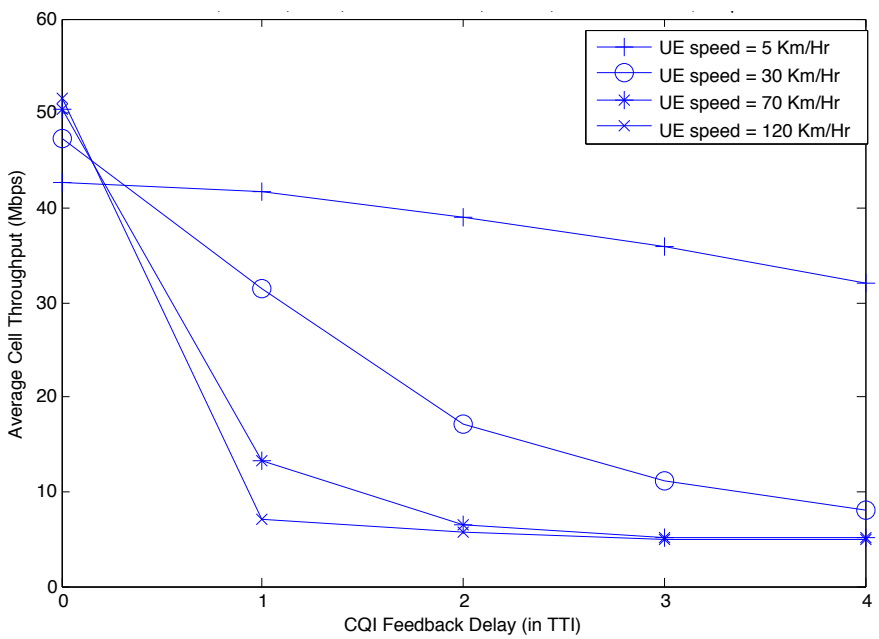

Fig. 8. Average Cell Throughput versus Feedback delay for proportional fair $(P F)$ scheduler

\section{CONCLUSions}

In this paper, we study the impact of UE speed and CQI uplink feedback delay on the throughput performance of an LTE network. By using standard compliance system level simulations, we have shown that in addition to CQI uplink delay the UE speed further degrades system performance. This happens due to the wrong selection of MCS as the CQI value use in the scheduling algorithm may not match the current channel conditions. We have studied the different scheduling algorithms and calculated average user throughput, average cell-edge user throughput and average cell throughput under different UE speed and uplink CQI feedback delay. We, finally, concluded that for an efficient scheduling algorithm at eNodeB, UE speed must be taken into account. The development of scheduling algorithm that takes into account the UE speed, estimate and correct potential delays in the feedback link is the scope of our future work.

\section{ACKNOWLEDGEMENT}

The research leading to these results was derived from the University of Greenwich Research \& Enterprise Investment Programme grant under agreement number RAE-ES-01/14.

\section{REFERENCES}

[1] M. Dehghani, K. Arshad, and R. MacKenzie, "Lte-advanced radio access enhancements: A survey," Wireless Personal Communications, pp. 1-31, 2014. [Online]. Available: http://dx.doi.org/10.1007/s11277$014-2062-y$

[2] T.-T. Tran, Y. Shin, and O.-S. Shin, "Overview of Enabling Technologies for 3GPP LTE-Advanced," EURASIP Journal on Wireless Communications and Networking, vol. 2012, no. 1, 2012. [Online]. Available: http://dx.doi.org/10.1186/1687-1499-2012-54

[3] J. C. Ikuno, M. Wrulich, and M. Rupp, "System Level Simulation of LTE Networks," in Proc. 2010 IEEE 71st Vehicular Technology Conference, Taipei, Taiwan, may 2010.

[4] D. H. Zarrinkoub, Overview of the LTE Physical Layer. John Wiley \& Sons, Ltd, 2014, pp. 13-46. [Online]. Available: http://dx.doi.org/10.1002/9781118443446.ch2

[5] R. Akl, S. Valentin, G. Wunder, and S. Stanczak, "Compensating for CQI Aging by Channel Prediction: The LTE Downlink," in 2012 IEEE Global Communications Conference (GLOBECOM), Dec 2012, pp. 4821-4827.

[6] Q.-T. Quoc-Tuan Vien and H. Nguyen, "CQI Reporting Strategies for Nonregenerative Two-way Relay Networks," in 2012 IEEE Wireless Communications and Networking Conference (WCNC), April 2012, pp. 974-979.

[7] J. Paris, M. Aguayo-Torres, and J. Entrambasaguas, "Non-ideal Adaptive Modulation: Bounded Signaling Information and Imperfect Adaptation," in IEEE Global Telecommunications Conference GLOBECOM'04, vol. 6, Nov 2004, pp. 3828-3834 Vol.6.

[8] L. Zhang, X. Ji, and M. Peng, "A Rank Adaptive Beamforming Scheme for TDD LTE System with Imperfect CSI," in 2012 International Conference on Wireless Communications Signal Processing (WCSP), Oct 2012, pp. 1-5.

[9] S. AlQahtani and M. Alhassany, "Comparing Different LTE Scheduling Schemes," in 2013 9th International Wireless Communications and Mobile Computing Conference (IWCMC), July 2013, pp. 264-269.

[10] "Evolved Universal Terrestrial Radio Access (EUTRA); Radio Frequency (RF) System Scenarios," http://www.3gpp.org/DynaReport/36942.htm, accessed: 2014-07-30. 